Original Article

\title{
The Clinical Outcomes of Different First-Line EGFR-TKIs Plus Bevacizumab in Advanced EGFR-Mutant Lung Adenocarcinoma
}

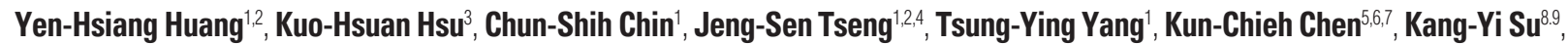 \\ Sung-Liang $\mathbf{Y u}^{8,9,10,11,12,13}$, Jeremy J.W. Chen ${ }^{2}$, Gee-Chen Chang ${ }^{2,45,6,7}$ \\ ${ }^{1}$ Division of Chest Medicine, Department of Internal Medicine, Taichung Veterans General Hospital, Taichung, ${ }^{2}$ Institute of Biomedical Sciences, \\ National Chung Hsing University, Taichung, ${ }^{3}$ Division of Critical Care and Respiratory Therapy, Department of Internal Medicine, Taichung Veterans \\ General Hospital, Taichung, ${ }^{4}$ Faculty of Medicine, School of Medicine, National Yang-Ming University, Taipei, ${ }^{5}$ Division of Pulmonary Medicine, \\ Department of Internal Medicine, Chung Shan Medical University Hospital, Taichung, ${ }^{6}$ Institute of Medicine, Chung Shan Medical University, \\ Taichung, ${ }^{7}$ School of Medicine, Chung Shan Medical University, Taichung, ${ }^{8}$ Department of Clinical Laboratory Sciences and Medical Biotechnology, \\ College of Medicine, National Taiwan University, Taipei, ${ }^{9}$ Department of Laboratory Medicine, National Taiwan University Hospital, Taipei, \\ ${ }^{10}$ Graduate Institute of Clinical Medicine, College of Medicine, National Taiwan University, Taipei, ${ }^{11}$ Center of Genomic and Precision Medicine, \\ National Taiwan University, Taipei, ${ }^{12}$ Institute of Medical Device and Imaging, College of Medicine, National Taiwan University, Taipei, \\ ${ }^{13}$ Graduate Institute of Pathology, College of Medicine, National Taiwan University, Taipei, Taiwan
}

Purpose The aim of this study was to investigate the efficacy of various epidermal growth factor receptor (EGFR)-tyrosine kinase inhibitors (TKIs) plus bevacizumab in advanced EGFR-mutant lung adenocarcinoma patients.

Materials and Methods From August 2016 to October 2020, we enrolled advanced lung adenocarcinoma patients harboring exon 19 deletion or L858R receiving gefitinib, erlotinib and afatinib plus bevacizumab as the first-line treatment for the purposes of analysis.

Results A total of 36 patients were included in the final analysis. Three patients received gefitinib, 17 received erlotinib, and 16 received afatinib combined with bevacizumab as the first-line treatment. The objective response rate was $77.8 \%$, and disease control rate was $94.4 \%$. The overall median progression-free survival (PFS) was 16.4 months, while the median PFS was 17.1 months in patients with exon 19 deletion, and 16.2 months in patients with L858R mutation ( $p=0.311$ ). Regarding the use of different EGFRTKIs, the median PFS was 17.1 months in the erlotinib group and 21.6 months in the afatinib group ( $p=0.617$ ). In patients with brain metastasis at baseline, the median PFS was 18.9 months in the erlotinib group and 16.4 months in the afatinib group ( $p=0.747$ ). Amongst patients harboring exon 19 deletion, the median PFS was 16.2 months in the erlotinib group and not-reached in the afatinib group $(p=0.141$ ). In patients with L858R mutation, the median PFS was 18.9 months in the erlotinib group and 16.2 months in the afatinib group $(p=0.481)$.

Conclusion Our research demonstrates that not only erlotinib combined with bevacizumab, but also afatinib plus bevacizumab as first-line treatment, provides solid clinical efficacy in advanced EGFR-mutant lung adenocarcinoma patients.

Key words ErbB receptors, Tyrosine kinase inhibitor, Bevacizumab, Lung neoplasms, Adenocarcinoma

\section{Introduction}

Lung cancer is the leading cause of mortality worldwide. Amongst patients with lung cancer, 80\%-85\% were classified as non-small cell lung cancer (NSCLC). Currently, the treatment for NSCLC is individualized and based on the results of histology and molecular biology tests. Patients with an oncogenic druggable mutation and genotype-directed therapy experience longer overall survival (OS) [1]. In Asia, epi- dermal growth factor receptor (EGFR) mutation is the most common driver mutation gene, and accounts for approximately 50\%-60\% of patients with lung adenocarcinoma [2,3]. Previous clinical trials and meta-analyses have demonstrated that EGFR-mutant advanced NSCLC patients undergoing first- and second-generation EGFR-tyrosine kinase inhibitor (TKI) treatment experienced better progression-free survival (PFS) and fewer adverse effects than patients who underwent platinum-based chemotherapy $[4,5]$.
Correspondence: Gee-Chen Chang

Division of Pulmonary Medicine, Department of Internal Medicine,

Chung Shan Medical University Hospital, No.110, Sec. 1, Jianguo N. Road,

Taichung, 402, Taiwan

Tel: 886-4-2473-9595 (ext. 34412) Fax: 886-4-2473-9595 (ext. 34710)

E-mail: geechen@gmail.com
Co-correspondence: Jeremy J.W. Chen

Institute of Biomedical Sciences, National Chung Hsing University, No. 145,

Xingda Rd., South Dist., Taichung, 402, Taiwan

Tel: 886-4-2284-0896 (ext. 125) Fax: 886-4-2285-3469

E-mail: jwchen@dragon.nchu.edu.tw 
Regarding different generations of EGFR-TKI, LUX-Lung 7 , a phase $2 \mathrm{~B}$ study, showed that afatinib, a second-generation EGFR-TKI, resulted in significantly greater improvement in PFS than gefitinib, a first-generation EGFR-TKI, in treatment-naive patients with EGFR-mutant NSCLC [6]. Another phase 3 trial, an ARCHER-1050 study, proved that EGFR-mutation-positive NSCLC patients given dacomitinib, a second-generation EGFR-TKI used as first-line treatment, experienced longer PFS and OS than did patients who were prescribed gefitinib [7]. Furthermore, the FLAURA study found that osimertinib, a third-generation EGFR-TKI, displayed efficacy superior to that of both gefitinib and erlotinib in the first-line treatment of EGFR mutation-positive advanced NSCLC $[8,9]$. Although we did not find any clinical trials to compare the efficacy of the second-generation and third-generation EGFR-TKIs, a retrospective study in Japan demonstrated that there was no significant difference in time to discontinuation between the afatinib and osimertinib groups [10].

To eradicate cancer cells, a series of stepwise events involving the anti-cancer immune response must be initiated [11]. Thus, a combination treatment was considered a reasonable approach to improve the efficacy of anti-cancer therapy and was investigated. In previous phase 2 clinical trials, the clinical efficacy of erlotinib with bevacizumab, a vascular endothelial growth factor (VEGF) pathway inhibitor, in NSCLC patients with activating EGFR mutation was inconclusive $[12,13]$. Three phase 3 trials, the NEJ 026, RELAY, and CTONG 1509 studies, proved that erlotinib plus VEGF pathway inhibitors (bevacizumab and ramucirumab) prolonged the PFS in NSCLC patients harboring exon 19 deletion and exon 21 L858R mutation [14-17]. Regarding the use of second-generation EGFR-TKIs combined with VEGF pathway inhibitors, a phase 2 trial, the AfaBev-CS study, remains ongoing for the purpose of evaluating the clinical benefits of afatinib combined with bevacizumab [18]. A multicenter observational study in Taiwan has found that the combination of afatinib and bevacizumab provides positive clinical outcomes for PFS and OS with acceptable safety results in untreated EGFR-mutated advanced lung adenocarcinoma patients [19].

Although there have been some real-world analyses of the efficacy of EGFR-TKIs plus bevacizumab [19-21], few studies have addressed the impact of different first-line EGFR-TKIs with bevacizumab on the clinical outcomes of combination therapy. Therefore, we conducted the present study in order to investigate the issues mentioned above.

\section{Materials and Methods}

\section{Study design and patients}

This study was a retrospective, single-center, observational study performed at Taichung Veterans General Hospital (TCVGH) in Taiwan.

We enrolled lung cancer patients between August 2016 and October 2020. To be eligible for the study, patients had to fulfill the following inclusion criteria: a diagnosis of histologically and cytologically confirmed adenocarcinoma, recurrence, or stage IV lung cancer according to the 8th edition of the American Joint Committee for Cancer staging system, activating EGFR mutation with exon 19 deletion or exon 21 L858R point mutation, treatment with first-line EGFR-TKIs, including gefitinib, erlotinib, and afatinib, as well as having received bevacizumab during EGFR-TKIs treatment. Patients were excluded if they had experienced EGFR mutations other than exon 19 deletion and L858R mutation, did not receive bevacizumab during EGFR-TKIs treatment, or if they had been diagnosed with another active malignancy. Computed tomography of the chest was performed every three months in order to qualify for National Health Insurance reimbursement. Additionally, for patients with brain metastasis, magnetic resonance imaging of the brain was conducted every three months. Treatment response to EGFR-TKIs plus bevacizumab was evaluated by the Response Evaluation Criteria in Solid Tumors (ver. 1.1).

Each patient's demographic and clinical data, including age, sex, smoking status, Eastern Cooperative Oncology Group performance status (ECOG PS), clinical stage, condition of brain metastasis at baseline, EGFR mutation status at baseline, type and starting dose of EGFR-TKI treatment, and starting dose of bevacizumab, as well as the best response to EGFR-TKI plus bevacizumab and best PFS and OS for EGFRTKI combined with bevacizumab were collected for analysis.

\section{EGFR mutation test}

The EGFR mutations of tumor tissue were assessed through use of either the Cobas EGFR Mutation Test v2 (Roche Molecular Systems, Pleasanton, CA) or matrix-assisted laser desorption ionization-time of flight mass spectrometry (MALDI-TOF MS). The method for MALDI-TOF MS was based upon our previous studies $[3,22,23]$. The detection procedure was according to the user's manual of the MassARRAY System (Cat. No.10411, SEQUENIM, San Diego, CA acquired by Agena Bioscience, http:// agenabio.com/, San Diego, CA in 2014). Extracted DNA was used to perform serial biochemical reactions, including 40 cycles of PCR reaction; shrimp alkaline phosphatase treatment and 200 cycles of signal nucleotide extension reaction by using the iPLEX Pro reagent kit containing Sequenase, iPLEX Pro reaction mixture, and 


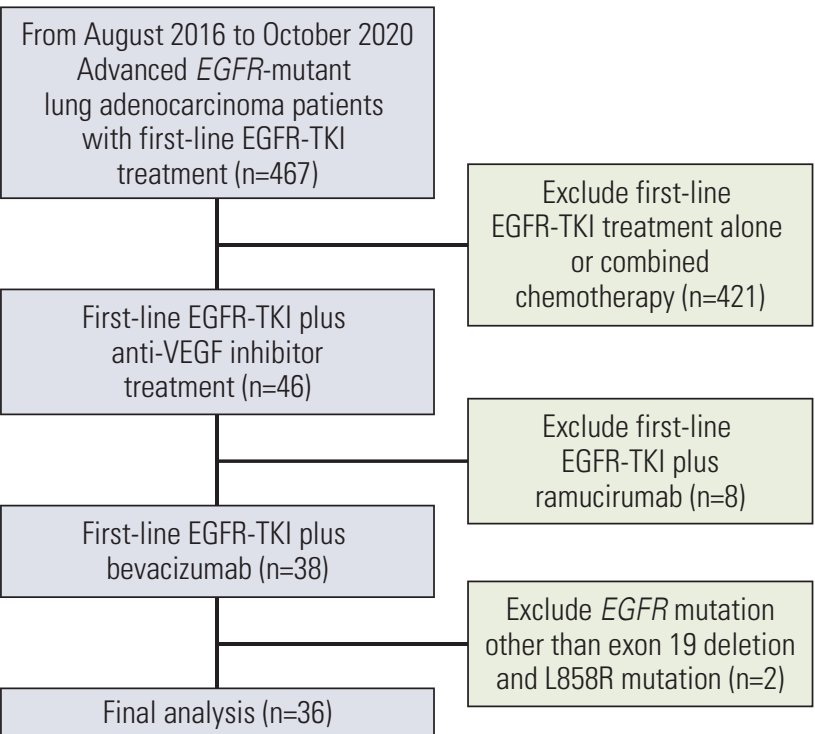

Fig. 1. The flowchart of patient collection. EGFR, epidermal growth factor receptor; TKI, tyrosine kinase inhibitor; VEGF, vascular endothelial growth factor.

home-designed probes. After SpectroClean Resin clean up, samples were loaded onto the matrix of the SpectroCHIP by Nanodispenser (Matrix) and then analyzed by the Bruker Autoflex MALDI-TOF MS. Data were collected and analyzed by MassARRAYTyper (ver. 4) software (Agena Bioscience).

\section{Statistical analyses}

Fisher's exact test was used to assess the difference between erlotinib and afatinib with regard to patients' characteristics. Survival curves, including PFS and OS, were estimated using the Kaplan-Meier method, while differences in survival time were analyzed using the log-rank test. All statistical tests were done with SPSS ver. 23.0 (IBM Corp., Armonk, NY) software. Two-tailed tests and p-values $<0.05$ for significance were used.

\section{Results}

\section{Baseline clinical characteristics of patients with first-line} EGFR-TKI combined with bevacizumab treatment $(n=36)$

In total, 36 recurrent and stage IV lung adenocarcinoma patients undergoing first-line EGFR-TKI combined with bevacizumab treatment were enrolled for analysis (Fig. 1). The baseline characteristics are shown in Table 1. The median age was 60 years old, with 17 male patients (47.2\%), and $27(75.0 \%)$ with no history of smoking. The ECOG PS was $0-1$ in all patients. Five patients $(13.9 \%)$ were stage
Table 1. The patients' characteristics and demographic data Characteristic

No. $(\%)(\mathrm{n}=36)$

\begin{tabular}{|c|c|}
\hline Age, median (range, yr) & $60(39-81)$ \\
\hline \multicolumn{2}{|l|}{ Sex } \\
\hline Male & $17(47.2)$ \\
\hline Female & $19(52.8)$ \\
\hline \multicolumn{2}{|l|}{ Smoking status } \\
\hline Non-smokers & $27(75.0)$ \\
\hline Former or current-smokers & $9(25.0)$ \\
\hline \multicolumn{2}{|l|}{ ECOG PS } \\
\hline 0 & $6(16.7)$ \\
\hline 1 & $30(83.3)$ \\
\hline \multicolumn{2}{|l|}{ Stage } \\
\hline Postoperation recurrence & $5(13.9)$ \\
\hline $4 \mathrm{~A}$ & $5(13.9)$ \\
\hline $4 \mathrm{~B}$ & $26(72.2)$ \\
\hline \multicolumn{2}{|l|}{ Brain metastasis at baseline } \\
\hline Yes & $22(61.1)$ \\
\hline No & $14(38.9)$ \\
\hline \multicolumn{2}{|l|}{ Baseline EGFR mutation status } \\
\hline Exon 19 deletion & $14(38.9)$ \\
\hline Exon 21 L858R point mutation & $22(61.1)$ \\
\hline \multicolumn{2}{|l|}{ First-line EGFR-TKI } \\
\hline Gefitinib & $3(8.3)$ \\
\hline Erlotinib & $17(47.2)$ \\
\hline Afatinib & $16(44.5)$ \\
\hline \multicolumn{2}{|l|}{ Treatment response } \\
\hline Stable disease & $6(16.7)$ \\
\hline Partial response & $28(77.7)$ \\
\hline Could not be evaluated & $2(5.6)$ \\
\hline Objective response rate $(\%)$ & 77.8 \\
\hline Disease control rate $(\%)$ & 94.4 \\
\hline
\end{tabular}

ECOG PS, Eastern Cooperative Oncology Group performance status; EGFR, epidermal growth factor receptor; TKI, tyrosine kinase inhibitor.

IVA, while $26(72.2 \%)$ were stage IVB. Five patients (13.9\%) experienced disease recurrence after surgery. Brain metastasis was noted at baseline in 22 patients $(61.1 \%)$, while fourteen (38.9\%) harbored EGFR exon 19 deletion, and 22 (61.1\%) had exon 21 L858R point mutation. Three patients (8.3\%) received gefitinib, $17(47.2 \%)$ received erlotinib and 16 $(44.5 \%)$ received afatinib as first-line EGFR-TKI plus bevacizumab treatment.

\section{Starting dose of EGFR-TKIs and bevacizumab $(n=36)$}

The median duration of treatment in patients with gefitin$\mathrm{ib}$, erlotinib and afatinib plus bevacizumab were 13.0 months (95\% confidence interval [CI], 5.3 to 20.7), 17.7 months (95\% CI, 12.4 to 23.0 ) and 22.3 months (95\% CI, 13.5 to 31.1), respectively. Three patients $(100 \%)$ received gefitinib as first- 
Table 2. The starting dose of EGFR-TKIs and bevacizumab

\section{Starting dose}

No. $(\mathrm{n}=36)$

\section{EGFR-TKI}

Gefitinib $250 \mathrm{mg}$

Erlotinib $150 \mathrm{mg} \quad 17$

Afatinib $40 \mathrm{mg} \quad 12$

Afatinib $30 \mathrm{mg}$

Bevacizumab $7.5 \mathrm{mg} / \mathrm{kg}$

Dose de-escalation

Erlotinib $150 \mathrm{mg} \rightarrow 100 \mathrm{mg}$

Afatinib $40 \mathrm{mg} \rightarrow 30 \mathrm{mg}$

$36^{\text {a) }}$

ECFR, epidermal growth factor receptor; TKL, tyrosine kinase inhibitor. ${ }^{a)}$ One patient discontinue bevacizumab due to proteinuria, ${ }^{b}$ Acneiform dermatitis, ${ }^{c}$ Acneiform dermatitis 2, stomatitis 1.
Table 3. The selective adverse events of EGFR-TKIs plus bevacizumab $(\mathrm{n}=36)$

\begin{tabular}{lrc} 
Adverse event & Any grade & Grade $\geq 3$ \\
Skin rash/Acne & $30(83.3)$ & $3(8.3)$ \\
Diarrhea & $16(44.4)$ & 0 \\
Paronychia & $16(44.4)$ & 0 \\
Proteinuria & $15(38.9)$ & $1(2.8)$ \\
Stomatitis & $13(36.1)$ & $1(2.8)$ \\
Increased aminotransferase & $11(30.6)$ & $3(8.3)$ \\
Hypertension & $5(13.9)$ & 0 \\
Bleeding & $4(11.1)$ & 0 \\
\hline
\end{tabular}

Values are presented as number (\%). EGFR, epidermal growth factor receptor; TKI, tyrosine kinase inhibitor.

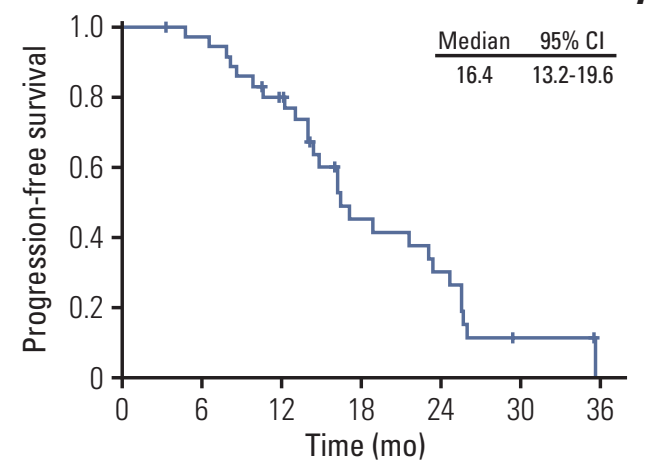

$\begin{array}{llllllll}\text { No. at risk } & 36 & 34 & 26 & 12 & 8 & 2 & 0\end{array}$

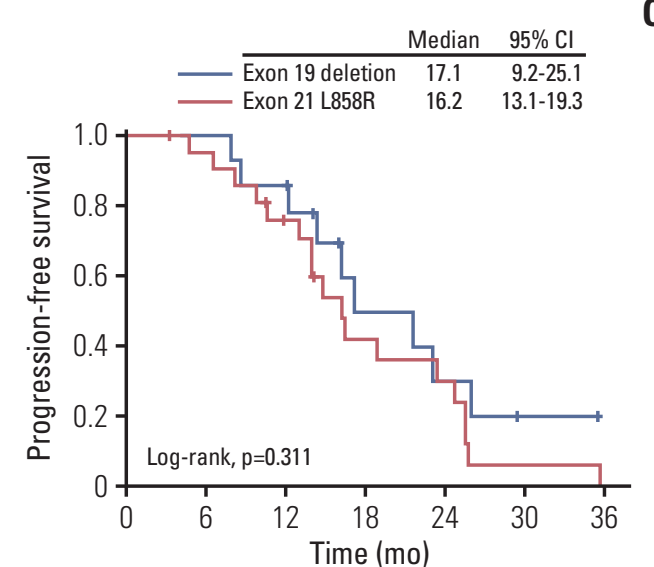

No. at risk

$\begin{array}{rlllllll}\text { Exon } 19 \text { deletion } & 14 & 14 & 12 & 5 & 3 & 1 & 0 \\ \text { Exon } 21 \text { L858R } & 22 & 20 & 14 & 7 & 5 & 1 & 0\end{array}$

A

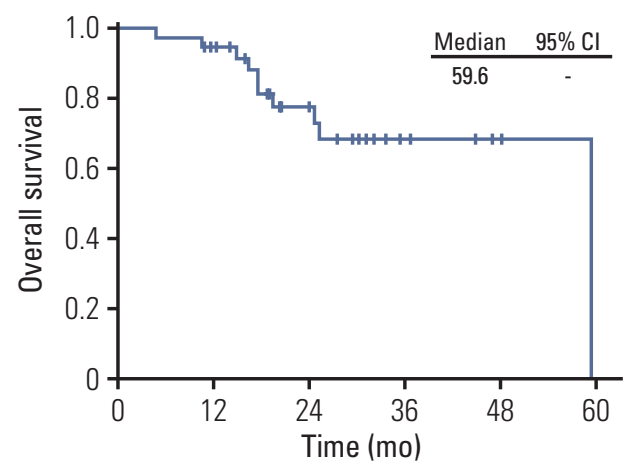

$\begin{array}{lllllll}\text { No. at risk } & 36 & 32 & 19 & 5 & 2 & 0\end{array}$

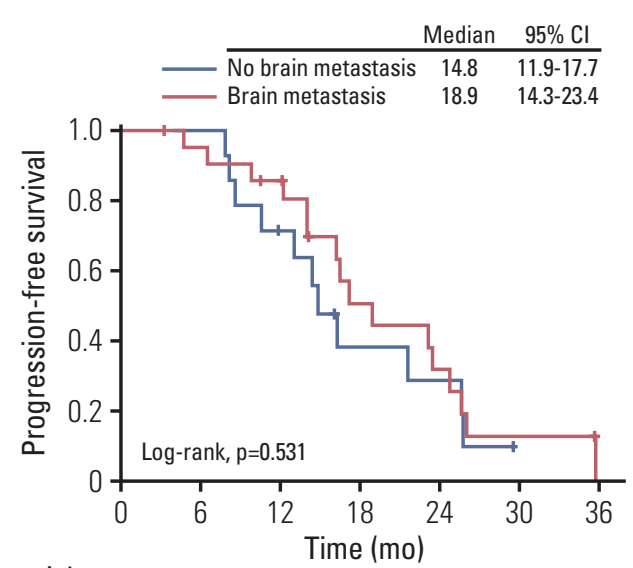

No. at risk

$\begin{array}{crrrrrrr}\text { No brain metastasis } & 14 & 14 & 9 & 4 & 3 & 0 & 0 \\ \text { Brain metastasis } & 22 & 20 & 17 & 8 & 5 & 2 & 0\end{array}$

Fig. 2. Clinical efficacy of epidermal growth factor receptor-tyrosine kinase inhibitor combined with bevacizumab. (A) Progression-free survival. (B) Overall survival. (C) Progression-free survival of patients with exon 19 deletion and exon 21 L858R mutation. (D) Progressionfree survival of patients with brain and without brain metastasis at baseline. CI, confidence interval. 
Table 4. The patients' characteristics of the erlotinib and afatinib groups $(n=33)$

\begin{tabular}{|c|c|c|c|}
\hline \multirow{2}{*}{ Characteristic } & \multicolumn{2}{|c|}{ First-line EGFR-TKI } & \multirow{2}{*}{ p-value } \\
\hline & Erlotinib & Afatinib & \\
\hline \multicolumn{4}{|l|}{ Age (yr) } \\
\hline$\geq 65$ & $7(41.2)$ & $4(25.0)$ & 0.465 \\
\hline$<65$ & $10(58.8)$ & $12(75.0)$ & \\
\hline \multicolumn{4}{|l|}{ Sex } \\
\hline Male & $9(52.9)$ & $7(43.8)$ & 0.732 \\
\hline Female & $8(47.1)$ & $9(56.2)$ & \\
\hline \multicolumn{4}{|l|}{ Smoking status } \\
\hline NS & $14(82.4)$ & $11(68.8)$ & 0.438 \\
\hline $\mathrm{C} / \mathrm{FS}$ & $3(17.6)$ & $5(31.2)$ & \\
\hline \multicolumn{4}{|l|}{ ECOG PS } \\
\hline 0 & $4(23.5)$ & $2(12.5)$ & 0.656 \\
\hline 1 & $13(76.5)$ & $14(87.5)$ & \\
\hline \multicolumn{4}{|l|}{ Stage } \\
\hline Postoperation recurrence & 0 & $4(25.0)$ & 0.096 \\
\hline $4 \mathrm{~A}$ & $2(11.8)$ & $1(6.2)$ & \\
\hline $4 \mathrm{~B}$ & $15(88.2)$ & $11(68.8)$ & \\
\hline \multicolumn{4}{|l|}{ Brain metastasis at baseline } \\
\hline Yes & $14(82.4)$ & $8(50.0)$ & 0.071 \\
\hline No & $3(17.6)$ & $8(50.0)$ & \\
\hline \multicolumn{4}{|l|}{ Bone metastasis at baseline } \\
\hline Yes & $12(70.6)$ & $12(75.0)$ & $>0.99$ \\
\hline No & $5(29.4)$ & $4(25.0)$ & \\
\hline \multicolumn{4}{|l|}{ Liver metastasis at baseline } \\
\hline Yes & 0 & $2(12.5)$ & 0.227 \\
\hline No & $17(100)$ & $14(87.5)$ & \\
\hline \multicolumn{4}{|l|}{ Baseline EGFR mutation } \\
\hline Exon 19 deletions & $7(41.2)$ & $6(37.5)$ & $>0.99$ \\
\hline Exon 21 L858R & $10(58.8)$ & $10(62.5)$ & \\
\hline
\end{tabular}

Values are presented as number (\%). C/FS, current/ former-smokers; ECOG PS, Eastern Cooperative Oncology Group performance status;

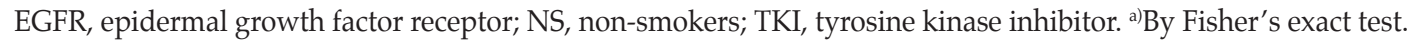

line EGFR-TKI treatment at a starting dose of $250 \mathrm{mg}$, while $17(100 \%)$ received erlotinib at a starting dose of $150 \mathrm{mg}$ (Table 2). Amongst the patients receiving afatinib as first-line therapy, $12(75 \%)$ took afatinib at a dosage of $40 \mathrm{mg}$, while four $(25 \%)$ started from $30 \mathrm{mg}$. Bevacizumab was administered at a dosage of $7.5 \mathrm{mg} / \mathrm{kg}$ in all patients $(100 \%)$ with median 15 cycles (ranging from 3 to 34 cycles). Four patients $(11.1 \%)$ decreased their dosage of EGFR-TKIs during treatment involving EGFR-TKI plus bevacizumab. Two patients with afatinib and one patient with erlotinib adjusted their dosage of EGFR-TKIs due to acneiform dermatitis (grade 3), and one patient with afatinib was adjusted because of stomatitis (grade 3). Additionally, one patient discontinued bevacizumab due to proteinuria (grade 3). The adverse events of EGFR-TKI plus bevacizumab were summarized in Table 3. The most common adverse event was skin rash and acne
$(83.3 \%)$, followed by diarrhea $(44.4 \%)$, paronychia $(44.4 \%)$, and proteinuria $(38.9 \%)$.

\section{Efficacy of first-line EGFR-TKI combined with bevaci- zumab $(\mathrm{n}=36)$}

The data cutoff date was 30 April 2021. The median follow-up time of PFS was 14.6 months, and the median follow-up time of OS was 24.2 months. Two patients' treatment response to first-line EGFR-TKI combined with bevacizumab could not be evaluated. Six patients had stable disease, and 28 patients had partial response under the strategy of EGFRTKI plus bevacizumab. The overall objective response rate (ORR) was $77.8 \%$, and the disease control rate (DCR) was 94.4\% (Table 1).

The estimated median PFS was 16.4 months (95\% CI, 13.2 to 19.6) (Fig. 2A), and the estimated median OS was 59.6 
A

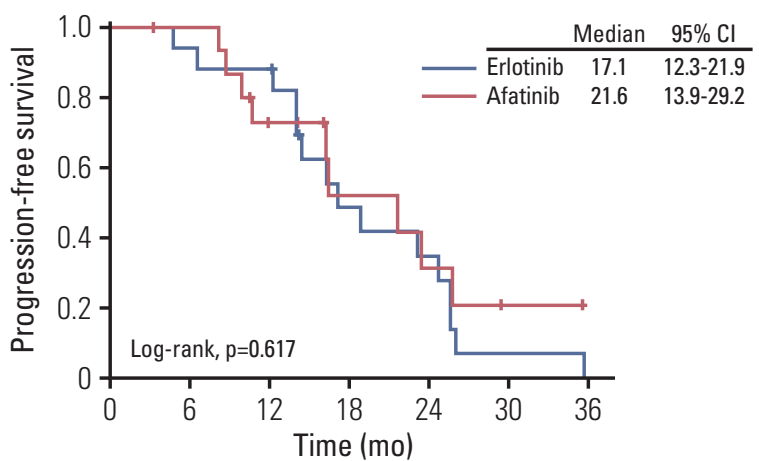

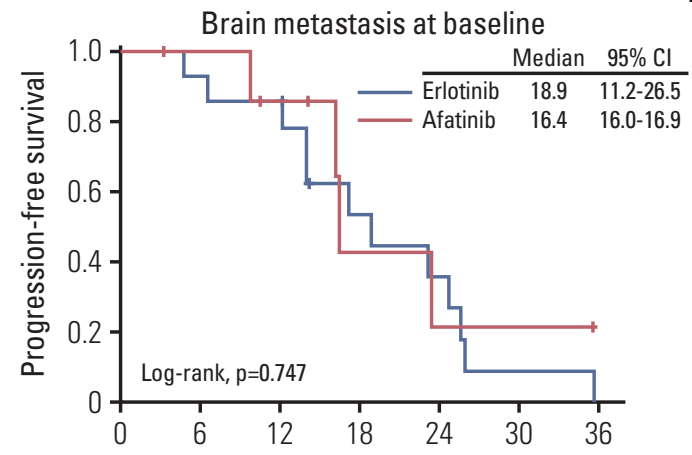

$\begin{array}{rrrrrrrr}\text { No. at risk } & & & & & & \\ \text { Erlotinib } & 17 & 16 & 15 & 7 & 5 & 1 & 0 \\ \text { Afatinib } & 16 & 15 & 9 & 5 & 3 & 1 & 0\end{array}$

$\begin{array}{llllllll}\text { Afatinib } & 16 & 15 & 9 & 5 & 3 & 1 & 0\end{array}$

C

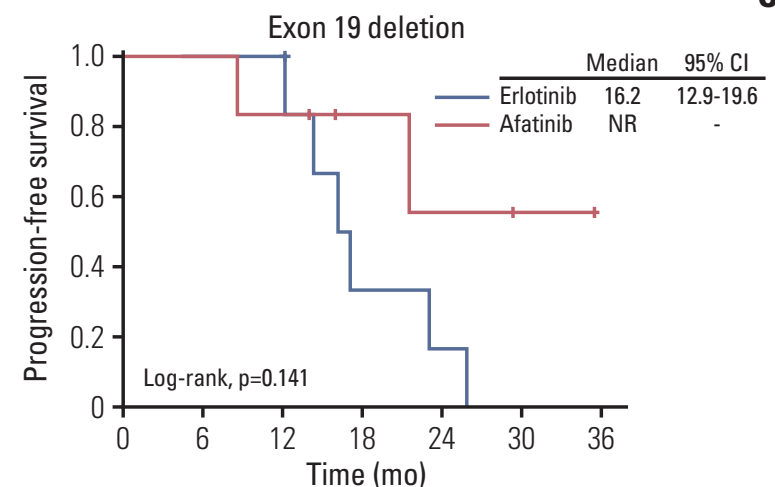

No. at risk

$\begin{array}{llllllll}\text { Erlotinib } & 7 & 7 & 7 & 2 & 1 & 0 & 0 \\ \text { Afatinib } & 6 & 6 & 5 & 3 & 2 & 1 & 0\end{array}$

$\begin{array}{rrrrrrrr}\text { No. at risk } & & & & & & & \\ \text { Erlotinib } & 14 & 13 & 12 & 6 & 4 & 1 & 0 \\ \text { Afatinib } & 8 & 7 & 5 & 2 & 1 & 1 & 0\end{array}$

D

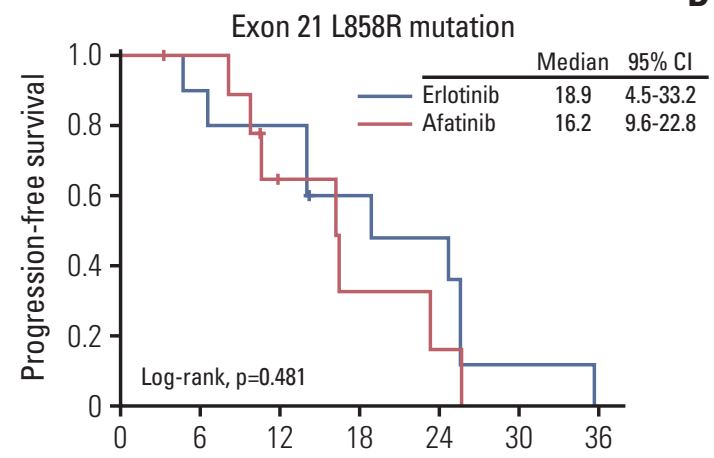

No. at risk

$\begin{array}{llllllll}\text { Erlotinib } & 10 & 9 & 8 & 5 & 4 & 1 & 0\end{array}$

$\begin{array}{llllllll}\text { Afatinib } & 10 & 9 & 4 & 2 & 1 & 0 & 0\end{array}$

Fig. 3. Progression-free survival of patients with erlotinib and afatinib as first-line treatment combined with bevacizumab. (A) Progression-free survival of different epidermal growth factor receptor-tyrosine kinase inhibitors (EGFR-TKIs) in all patients. (B) Progressionfree survival of different EGFR-TKIs in patients with brain metastasis at baseline. (C) Progression-free survival of different EGFR-TKIs in patients harboring exon 19 deletion. (D) Progression-free survival of different EGFR-TKIs in patients harboring exon 21 L858R point mutation. CI, confidence interval.

months (Fig. 2B). Concerning different EGFR mutations, in patients with exon 19 deletion, the estimated median PFS was 17.1 months (95\% CI, 9.2 to 25.1), with the estimated median PFS being 16.2 months (95\% CI, 13.1 to 19.3) amongst patients harboring L858R mutation (log-rank test, $\mathrm{p}=0.311$ ) (Fig. 2C). In patients with brain metastasis at baseline, the estimated median PFS was 18.9 months (95\% CI, 14.3 to 23.4), and the estimated median PFS was 14.8 months (95\% CI, 11.9 to 17.7) amongst patients without brain metastasis at baseline (log-rank test, $\mathrm{p}=0.531$ ) (Fig. 2D).

\section{Comparison of patients' characteristics between the erlo- tinib and afatinib groups $(n=33)$}

After excluding patients who were administered gefitinib as first-line EGFR-TKI plus bevacizumab treatment, 33 patients given erlotinib or afatinib combined with bevacizum$\mathrm{ab}$ as first-line treatment were enrolled for analysis. Comparisons of patients' baseline characteristics between the erlotinib $(n=17)$ and afatinib groups $(n=16)$ are summarized in Table 4. Although there were no statistical differences, the percentage of patients with brain metastasis at baseline was higher in the erlotinib group than in the afatinib group. All four patients experiencing recurrence after surgery received afatinib plus bevacizumab as first-line treatment. Other clinical factors 
including age, sex, smoking status, ECOG PS, baseline EGFR mutation, bone metastasis at baseline, and liver metastasis at baseline were equally distributed in the erlotinib and afatinib groups.

\section{Comparison of PFS between the erlotinib and afatinib groups $(n=33)$}

The estimated median PFS of patients receiving erlotinib plus bevacizumab was 17.1 months (95\% CI, 12.3 to 21.9), and the estimated median PFS of patients with afatinib plus bevacizumab was 21.6 months (95\% CI, 13.9 to 29.2) (logrank test, $\mathrm{p}=0.617$ ) (Fig. 3A). Amongst patients with brain metastasis at baseline, the estimated median PFS of patients in the erlotinib group was 18.9 months (95\% CI, 11.2 to 26.5), and the estimated median PFS of patients in the afatinib group was 16.4 months (95\% CI, 16.0 to 16.9) (log-rank test, $\mathrm{p}=0.747$ ) (Fig. 3B).

Regarding different EGFR mutations at baseline, in patients with exon 19 deletion, the estimated median PFS of patients in the erlotinib group was 16.2 months $(95 \%$ CI, 12.9 to 19.6), while the estimated median PFS of patients in the afatinib group was not reached (log-rank test, $\mathrm{p}=0.141$ ) (Fig. 3C). In patients with exon 21 L858R point mutation, the estimated median PFS of patients in the erlotinib group was 18.9 months (95\% CI, 4.5 to 33.2), while the estimated median PFS of patients in the afatinib group was 16.2 months ( $95 \%$ CI, 9.6 to 22.8) (log-rank test, $\mathrm{p}=0.481$ ) (Fig. 3D).

\section{Discussion}

Our research has demonstrated that in real-world practice EGFR-TKIs combined with bevacizumab as first-line treatment provided satisfactory efficacy in advanced lung adenocarcinoma patients harboring EGFR mutation. No matter the subtype of EGFR mutation or status of brain metastasis at baseline, patients could experience clinical benefits from the combination treatment of EGFR-TKI plus bevacizumab. The statistical analysis proved that there was no difference in PFS between the afatinib and erlotinib combined with bevacizumab therapies.

In the clinical trial of NEJ026, the ORR and DCR of erlotinib plus bevacizumab for patients with EGFR-positive NSCLC were $72 \%$ and $95 \%$, respectively [14]. Additionally, the median PFS of the erlotinib plus bevacizumab group was significantly longer than that of the erlotinib group (16.9 vs. 13.3 months; hazard ratio [HR], $0.605 ; 95 \%$ CI, 0.417 to 0.877 ; $\mathrm{p}=0.016$ ). Wang et al. [20] conducted a real-world, singlearm study which showed that the combination of erlotinib and bevacizumab resulted in an ORR of $83.7 \%$ and a DCR of 97.9\% for untreated metastatic EGFR-mutated lung adeno- carcinoma. The median PFS was 22.0 months (95\% CI, 19.7 to 22.33), and the median OS 47.6 months (95\% CI, 38.87 to 56.37). In another real-world, two-arm study, Tsai et al. [21] demonstrated that the combination of EGFR-TKI and bevacizumab improved PFS when compared to the monotherapy group (17.0 months vs. 11.0 months; HR, $0.48 ; \mathrm{p}=0.002$ ) for patients with EGFR mutation-positive advanced NSCLC. In our research, the ORR was $77.8 \%$, and the DCR $94.4 \%$ in advanced EGFR-mutant lung adenocarcinoma patients receiving EGFR-TKI plus bevacizumab. The median PFS was 16.4 months, and the median OS 59.6 months. Taken together, the abovementioned clinical trials and other real-world studies, along with the present study confirm the clinical efficacy of the combination therapy using EGFR-TKI plus bevacizumab.

At present, the clinical benefits of afatinib plus bevacizum$a b$ versus afatinib alone have not yet been evaluated through clinical trials. In a phase 1 single-arm study, afatinib plus bevacizumab provided an ORR of $81.3 \%$ and DCR of $100 \%$ for untreated patients with advanced EGFR-mutant NSCLC, where the median PFS was 24.2 months (95\% CI, 16.3 to NR) [24,25]. Another phase 1 study showed that EGFR-TKI-naïve advanced non-squamous NSCLC patients harboring EGFR mutations receiving afatinib combined with bevacizumab treatment had an ORR of $55 \%$ and DCR of $100 \%$, where the median PFS was 16.8 months (95\% CI, 4.4 to 18.8) [26]. A multicenter observational study demonstrated that the ORRs and DCRs of afatinib plus bevacizumab therapy were $87.7 \%$ and $100 \%$, respectively, for patients with untreated EGFRmutated advanced lung adenocarcinoma [19]. Regarding the above study, the median PFS was 23.9 months (95\% CI, 17.56 to 29.17), and the median OS was 45.9 months (95\% CI, 39.50 to 53.60). Furthermore, Hsu et al. [19] stated that most of the adverse events were grade 2 or lower and manageable, with $35.4 \%$ (17/48) experiencing dose reduction from $40 \mathrm{mg}$ to $30 \mathrm{mg}$. In our study, the median PFS was 21.6 months (95\% CI, 13.9 to 29.2) in advanced EGFR-mutant lung adenocarcinoma patients who were administered afatinib plus bevacizumab as first-line treatment. Three patients $(25 \%)$ had dose de-escalation from $40 \mathrm{mg}$ to $30 \mathrm{mg}$ (due to acneiform dermatitis in two patients, and stomatitis in 1 patient). Although Hsu's study and the present investigation demonstrated the clinical efficacy of afatinib combined with bevacizumab, clinical trials are still necessary in order to confirm whether the combination therapy of afatinib and bevacizumab could provide better clinical outcomes than afatinib alone.

Previous meta-analysis studies showed that first-generation and second-generation EGFR-TKIs statistically prolonged PFS in a significant manner when compared with platinum-based chemotherapy [4,5]. However, it seems that patients with exon 19 deletion experience better clini- 
cal benefits through EGFR-TKIs treatment than do patients with L858R point mutation based on a relatively lower HR. This phenomenon could be explained by the difference in structure of EGFR, the rate of concomitant mutation, and tumor mutation burden between exon 19 deletion and exon 21 L858R point mutation [27-29]. In combination therapy involving EGFR-TKIs and anti-angiogenic agents, the NEJ026 showed that the HR of PFS and OS was relatively lower in patients harboring L858R mutation than in patients with exon 19 deletion [14,15]. The HRs of PFS and OS for patients harboring L858R mutation were 0.57 and 0.79 , respectively. The HR of PFS in patient with exon 19 deletion was 0.69 , and the HR of OS was 1.34. In the RELAY study, the HRs of PFS in the L858R mutation and exon 19 deletion groups were 0.618 and 0.651 , respectively [16]. In a real-world analysis, Tsai et al. [21] suggested that the combination of EGFR-TKI and bevacizumab could significantly improve PFS and OS in NSCLC patients harboring L858R mutation group; however, the subgroup analysis found that there was no clinical efficacy in patients with exon 19 deletion. In our research, there was no statistical difference in the median PFS of EGFR-TKIs plus bevacizumab between patients with exon 19 deletion and L858R mutation (17.1 months vs. 16.2 months; log-rank test, $\mathrm{p}=0.311$ ). According to the above results, this implies that EGFR-TKIs combined with VEGF inhibitors could overcome the weak effect achieved using EGFR-TKI alone in patients harboring L858R point mutation.

The clinical trials of LUX-Lung 7 and ARCHER-1050 showed that second-generation EGFR-TKIs resulted in significantly greater improvement in PFS compared with gefitinib in treatment-naive patients with EGFR-mutant NSCLC [6,7]. In real-world research, Kim et al. [30] demonstrated that the PFS of afatinib was significantly longer than that of both gefitinib and erlotinib (19.1 months vs. 13.7 vs. 14.0 months, $\mathrm{p}=0.001$ ). However, no study has ever been performed to investigate the clinical efficacy of different EGFR-TKIs plus bevacizumab. In the present study, we have shown that the median PFS of patients administered erlotinib plus bevacizumab treatment was 17.1 months $(95 \%$ CI, 12.3 to 21.9), and the median PFS of patients receiving afatinib plus bevacizumab was 21.6 months (95\% CI, 13.9 to 29.2). Although there was a trend indicating that the patients in afatinib group had longer PFS than the erlotinib group, it did not reach statistical significance (log-rank test, $\mathrm{p}=0.617$ ). Regarding the subtype of EGFR mutation at baseline and the status of brain metastasis at baseline, there was no significant difference between the erlotinib and afatinib groups. Based on our findings, we suggest that the patients given afatinib plus bevacizumab as first-line treatment experienced good clinical efficacy, which was not inferior to patients receiving erlotinib plus bevacizumab.
Recent clinical trials have investigated the role of thirdgeneration EGFR-TKI combined with anti-VEGF inhibitor [31-33]. In a phase 1 study, Yu et al. [31] demonstrated that 25 advanced T790M-positive EGFR-mutant NSCLC patients receiving osimertinib plus ramucirumab experienced an ORR of $76 \%$ and a median PFS of 11.0 months with tolerable toxicity. However, WJOG $8715 \mathrm{~L}$, a randomized phase 2 trial, revealed that osimertinib plus bevacizumab did not provide better PFS than osimertinib alone in EGFR T790Mmutated NSCLC patients previously treated with EGFR-TKI [32]. In a first-line setting, a phase $1 / 2$ trial enrolled 49 metastatic EGFR-mutant lung cancer patients who received the combination treatment of osimertinib and bevacizumab [33]. The PFS at 12 months was $76 \%$, and the median PFS was 19 months. Although osimertinib is a more potent EGFR-TKI, we still need further clinical trials to confirm the clinical benefits of osimertinib plus anti-VEGF inhibitor in advanced EGFR-mutant NSCLC.

Previously studies have shown that approximately 50\%$60 \%$ of EGFR-mutant NSCLC patients harbor T790M mutation after progressive disease following first-line EGFR-TKIs treatment [34,35]. In our study, $27(75 \%)$ patients experienced disease progression at the end of the follow-up period. Fourteen $(51.8 \%)$ of them received re-biopsy, and $8(57 \%)$ patients harbored T790M mutation. Previous clinical trials and real-world data have also confirmed that the combination treatment of EGFR-TKI and anti-angiogenic agents did not influence the frequency of T790M mutation [16,19,21]. In a FLAURA study, NSCLC patients harboring EGFR mutation and receiving osimertinib as first-line treatment experienced longer PFS and OS than patients with first-generation EGFRTKIs $[8,9]$. Alternatively, the GioTag study demonstrated that the median time on treatment with afatinib and sequential osimertinib was 27.7 months, while the median OS was 37.6 months in patients with EGFR-mutation-positive NSCLC [36]. In the present study, 6 patients with T790M mutation received EGFR-TKI plus bevacizumab, with subsequent osimertinib treatment, resulting in an estimated PFS and OS of 32.5 and 59.6 months, respectively. Thus, if further research is able to prove that the PFS of first- and second-generation EGFR-TKIs plus bevacizumab is not inferior to that of osimertinib in EGFR-mutant NSCLC patients, the combination therapy of EGFR-TKI and VEGF pathway inhibitors may be a better choice as a first-line treatment.

The present research is the first study to investigate the impact of different EGFR-TKIs on the clinical efficacy of combination therapy with bevacizumab in advanced EGFR-mutant lung adenocarcinoma patients. However, this study has still some limitations. First, because bevacizumab is not reimbursed by National Health Insurance in Taiwan, the group of patients given EGFR-TKI plus bevacizumab as 
first-line treatment was relatively small. Second, the present research was a single-center, retrospective study, so there may have been greater bias when compared with prospectively designed studies. Third, only Taiwanese individuals were eligible for analysis. Therefore, our findings may not be generalizable to other ethnic populations. Finally, due to economic considerations, our patients received bevacizumab with a dose of $7.5 \mathrm{mg} / \mathrm{kg}$ rather than the standard dose of 15 $\mathrm{mg} / \mathrm{kg}$ used in clinical trials. However, previous clinical trials have demonstrated that non-squamous NSCLC patients receiving $7.5 \mathrm{mg} / \mathrm{kg}$ bevacizumab with chemotherapy experienced clinical efficacy equal to that of patients receiving 15 $\mathrm{mg} / \mathrm{kg}$ bevacizumab $[37,38]$. Thus, we believe that the use of EGFR-TKIs plus a reduced dose of bevacizumab likely did not influence our findings.

Our findings shed light on the clinical efficacy of EGFRTKI plus bevacizumab as a first-line treatment in advanced EGFR-mutant lung adenocarcinoma patients, as well as the impact of different EGFR-TKIs used, in terms of the clinical outcomes of combination therapy with bevacizumab. In conclusion, our research demonstrated that patients with combination therapy of EGFR-TKI and bevacizumab experienced satisfactory PFS and did not have a decreased mutation rate of T790M mutation. Additionally, we suggest that not only erlotinib combined with bevacizumab, but also afatinib plus bevacizumab as first-line treatment, provided sufficient efficacy in advanced EGFR-mutant lung adenocarcinoma patients.

\section{Ethical Statement}

The study was approved by the Institutional Review Board (IRB) of TCVGH, Taiwan, with written informed consent for genetic testing and clinical data records obtained from all patients (IRB No. CF12019).

\section{Author Contributions}

Conceived and designed the analysis: Huang $\mathrm{YH}$, Hsu $\mathrm{KH}$, Chin CS, Tseng JS, Yang TY, Chen KC, Su KY, Yu SL, Chen JJW, Chang GC.

Collected the data: Huang YH, Hsu KH, Chin CS, Tseng JS, Yang TY, Chen KC, Su KY, Yu SL, Chen JJW, Chang GC.

Contributed data or analysis tools: Huang $\mathrm{YH}, \mathrm{Hsu} \mathrm{KH}$, Chin CS, Tseng JS, Yang TY, Chen KC, Su KY, Yu SL, Chen JJW, Chang GC. Performed the analysis: Huang $\mathrm{YH}$, Hsu KH, Chin CS, Tseng JS, Yang TY, Chen KC, Su KY, Yu SL, Chen JJW, Chang GC.

Wrote the paper: Huang YH, Hsu KH, Chin CS, Tseng JS, Yang TY, Chen KC, Su KY, Yu SL, Chen JJW, Chang GC.

\section{Conflicts of Interest}

Conflict of interest relevant to this article was not reported.

\section{References}

1. Kris MG, Johnson BE, Berry LD, Kwiatkowski DJ, Iafrate AJ, Wistuba, II, et al. Using multiplexed assays of oncogenic drivers in lung cancers to select targeted drugs. JAMA. 2014;311:1998-2006.

2. Shi Y, Au JS, Thongprasert S, Srinivasan S, Tsai CM, Khoa $\mathrm{MT}$, et al. A prospective, molecular epidemiology study of EGFR mutations in Asian patients with advanced non-smallcell lung cancer of adenocarcinoma histology (PIONEER). J Thorac Oncol. 2014;9:154-62.

3. Hsu KH, Ho CC, Hsia TC, Tseng JS, Su KY, Wu MF, et al. Identification of five driver gene mutations in patients with treatment-naive lung adenocarcinoma in Taiwan. PLoS One. 2015;10:e0120852.

4. Kuan FC, Kuo LT, Chen MC, Yang CT, Shi CS, Teng D, et al. Overall survival benefits of first-line EGFR tyrosine kinase inhibitors in EGFR-mutated non-small-cell lung cancers: a systematic review and meta-analysis. Br J Cancer. 2015;113:151928.

5. Lee CK, Davies L, Wu YL, Mitsudomi T, Inoue A, Rosell R, et al. Gefitinib or erlotinib vs chemotherapy for EGFR mutationpositive lung cancer: individual patient data meta-analysis of overall survival. J Natl Cancer Inst. 2017;109:djw279.

6. Park K, Tan EH, O’Byrne K, Zhang L, Boyer M, Mok T, et al.
Afatinib versus gefitinib as first-line treatment of patients with EGFR mutation-positive non-small-cell lung cancer (LUX-Lung 7): a phase 2B, open-label, randomised controlled trial. Lancet Oncol. 2016;17:577-89.

7. Wu YL, Cheng Y, Zhou X, Lee KH, Nakagawa K, Niho S, et al. Dacomitinib versus gefitinib as first-line treatment for patients with EGFR-mutation-positive non-small-cell lung cancer (ARCHER 1050): a randomised, open-label, phase 3 trial. Lancet Oncol. 2017;18:1454-66.

8. Soria JC, Ohe Y, Vansteenkiste J, Reungwetwattana T, Chewaskulyong B, Lee $\mathrm{KH}$, et al. Osimertinib in untreated EGFR-mutated advanced non-small-cell lung cancer. N Engl J Med. 2018;378:113-25.

9. Ramalingam SS, Vansteenkiste J, Planchard D, Cho BC, Gray JE, Ohe $Y$, et al. Overall survival with osimertinib in untreated, EGFR-mutated advanced NSCLC. N Engl J Med. 2020;382:41-50.

10. Ito K, Morise M, Wakuda K, Hataji O, Shimokawaji T, Takahashi $\mathrm{K}$, et al. A multicenter cohort study of osimertinib compared with afatinib as first-line treatment for EGFR-mutated non-small-cell lung cancer from practical dataset: CJLSG1903. ESMO Open. 2021;6:100115.

11. Chen DS, Mellman I. Oncology meets immunology: the can- 
cer-immunity cycle. Immunity. 2013;39:1-10.

12. Stinchcombe TE, Janne PA, Wang X, Bertino EM, Weiss J, B zhenova L, et al. Effect of erlotinib plus bevacizumab vs erlotinib alone on progression-free survival in patients with advanced EGFR-mutant non-small cell lung cancer: a phase 2 randomized clinical trial. JAMA Oncol. 2019;5:1448-55.

13. Seto T, Kato T, Nishio M, Goto K, Atagi S, Hosomi Y, et al. Erlotinib alone or with bevacizumab as first-line therapy in patients with advanced non-squamous non-small-cell lung cancer harbouring EGFR mutations (JO25567): an openlabel, randomised, multicentre, phase 2 study. Lancet Oncol. 2014;15:1236-44.

14. Saito H, Fukuhara T, Furuya N, Watanabe K, Sugawara S, Iwasawa $S$, et al. Erlotinib plus bevacizumab versus erlotinib alone in patients with EGFR-positive advanced non-squamous non-small-cell lung cancer (NEJ026): interim analysis of an open-label, randomised, multicentre, phase 3 trial. Lancet Oncol. 2019;20:625-35.

15. Maemondo M, Fukuhara T, Saito H, Furuya N, Watanabe $\mathrm{K}$, Sugawara S, et al. NEJ026: final overall survival analysis of bevacizumab plus erlotinib treatment for NSCLC patients harboring activating EGFR-mutations. J Clin Oncol. 2020;38:15(Suppl):9506.

16. Nakagawa K, Garon EB, Seto T, Nishio M, Ponce Aix S, PazAres L, et al. Ramucirumab plus erlotinib in patients with untreated, EGFR-mutated, advanced non-small-cell lung cancer (RELAY): a randomised, double-blind, placebo-controlled, phase 3 trial. Lancet Oncol. 2019;20:1655-69.

17. Zhou Q, Wu YL, Cheng Y, Liu Y, Chen G, Cui J, et al. CTONG 1509: phase III study of bevacizumab with or without erlotinib in untreated Chinese patients with advanced EGFRmutated NSCLC. Ann Oncol. 2019;30(Suppl 5):v603.

18. Ninomiya T, Ishikawa N, Inoue K, Kubo T, Yasugi M, Shibayama $\mathrm{T}$, et al. Phase 2 study of afatinib alone or combined with bevacizumab in chemonaive patients with advanced nonsmall-cell lung cancer harboring EGFR mutations: AfaBev-CS study protocol. Clin Lung Cancer. 2019;20:134-8.

19. Hsu PC, Huang CY, Wang CC, Kuo SC, Chu CH, Tung PH, et al. The combination of afatinib and bevacizumab in untreated EGFR-mutated advanced lung adenocarcinoma: a multicenter observational study. Pharmaceuticals (Basel). 2020;13:331.

20. Wang CC, Chiu LC, Tung PH, Kuo SC, Chu CH, Huang AC, et al. A real-world analysis of patients with untreated metastatic epidermal growth factor receptor (EGFR)-mutated lung adenocarcinoma receiving first-line erlotinib and bevacizumab combination therapy. Oncol Ther. 2021;9:489-503.

21. Tsai JS, Su PL, Yang SC, Chang CC, Lin CY, Yen YT, et al. EGFR-TKI plus bevacizumab versus EGFR-TKI monotherapy for patients with EGFR mutation-positive advanced non-small cell lung cancer: a propensity score matching analysis. J Formos Med Assoc. 2021;120:1729-39.

22. Tsai TH, Su KY, Wu SG, Chang YL, Luo SC, Jan IS, et al. RNA is favourable for analysing EGFR mutations in malignant pleural effusion of lung cancer. Eur Respir J. 2012;39:677-84.

23. Su KY, Chen HY, Li KC, Kuo ML, Yang JC, Chan WK, et al. Pretreatment epidermal growth factor receptor (EGFR) T790M mutation predicts shorter EGFR tyrosine kinase inhibitor response duration in patients with non-small-cell lung cancer. J Clin Oncol. 2012;30:433-40.

24. Ninomiya T, Nogami N, Kozuki T, Harada D, Kubo T, Ohashi $\mathrm{K}$, et al. A phase I trial of afatinib and bevacizumab in chemonaive patients with advanced non-small-cell lung cancer harboring EGFR mutations: Okayama Lung Cancer Study Group Trial 1404. Lung Cancer. 2018;115:103-8.

25. Ninomiya T, Nogami N, Kozuki T, Harada D, Kubo T, Ohashi $\mathrm{K}$, et al. Updated analysis of a phase I trial of afatinib (Afa) and bevacizumab (Bev) in chemo-naïve patients (pts) with advanced non-small cell lung cancer (NSCLC) harboring EGFR-mutations: OLCSG1404. Ann Oncol. 2019;30(Suppl 5):v626.

26. Ko R, Shukuya T, Imamura CK, Tokito T, Shimada N, Koyama R, et al. Phase I study of afatinib plus bevacizumab in patients with advanced non-squamous non-small cell lung cancer harboring EGFR mutations. Transl Lung Cancer Res. 2021;10:183-92.

27. Harrison PT, Vyse S, Huang PH. Rare epidermal growth factor receptor (EGFR) mutations in non-small cell lung cancer. Semin Cancer Biol. 2020;61:167-79.

28. Hong S, Gao F, Fu S, Wang Y, Fang W, Huang Y, et al. Concomitant genetic alterations with response to treatment and epidermal growth factor receptor tyrosine kinase inhibitors in patients with EGFR-mutant advanced non-small cell lung cancer. JAMA Oncol. 2018;4:739-42.

29. Jiao XD, He X, Qin BD, Liu K, Wu Y, Liu J, et al. The prognostic value of tumor mutation burden in EGFR-mutant advanced lung adenocarcinoma, an analysis based on cBioPortal data base. J Thorac Dis. 2019;11:4507-15.

30. Kim Y, Lee SH, Ahn JS, Ahn MJ, Park K, Sun JM. Efficacy and safety of afatinib for EGFR-mutant non-small cell lung cancer, compared with gefitinib or erlotinib. Cancer Res Treat. 2019;51:502-9.

31. Yu HA, Paz-Ares LG, Yang JC, Lee KH, Garrido P, Park K, et al. Phase I study of the efficacy and safety of ramucirumab in combination with osimertinib in advanced T790M-positive EGFR-mutant non-small cell lung cancer. Clin Cancer Res. 2021;27:992-1002.

32. Akamatsu H, Toi Y, Hayashi H, Fujimoto D, Tachihara M, Furuya N, et al. Efficacy of osimertinib plus bevacizumab vs osimertinib in patients with EGFR T790M-mutated non-small cell lung cancer previously treated with epidermal growth factor receptor-tyrosine kinase inhibitor: West Japan Oncology Group 8715L phase 2 randomized clinical trial. JAMA Oncol. 2021;7:386-94.

33. Yu HA, Schoenfeld AJ, Makhnin A, Kim R, Rizvi H, Tsui D, et al. Effect of osimertinib and bevacizumab on progressionfree survival for patients with metastatic EGFR-mutant lung cancers: a phase $1 / 2$ single-group open-label trial. JAMA Oncol. 2020;6:1048-54.

34. Yu HA, Arcila ME, Rekhtman N, Sima CS, Zakowski MF, Pao $\mathrm{W}$, et al. Analysis of tumor specimens at the time of acquired resistance to EGFR-TKI therapy in 155 patients with EGFRmutant lung cancers. Clin Cancer Res. 2013;19:2240-7.

35. Huang YH, Hsu KH, Tseng JS, Chen KC, Hsu CH, Su KY, et 
al. The association of acquired T790M mutation with clinical characteristics after resistance to first-line epidermal growth factor receptor tyrosine kinase inhibitor in lung adenocarcinoma. Cancer Res Treat. 2018;50:1294-303.

36. Hochmair MJ, Morabito A, Hao D, Yang CT, Soo RA, Yang JC, et al. Sequential afatinib and osimertinib in patients with EGFR mutation-positive non-small-cell lung cancer: final analysis of the GioTag study. Future Oncol. 2020;16:2799-808.

37. Reck M, von Pawel J, Zatloukal P, Ramlau R, Gorbounova V, Hirsh V, et al. Phase III trial of cisplatin plus gemcitabine with either placebo or bevacizumab as first-line therapy for nonsquamous non-small-cell lung cancer: AVAil. J Clin Oncol. 2009;27:1227-34.

38. Mok TS, Hsia TC, Tsai CM, Tsang K, Chang GC, Chang JW, et al. Efficacy of bevacizumab with cisplatin and gemcitabine in Asian patients with advanced or recurrent non-squamous non-small cell lung cancer who have not received prior chemotherapy: a substudy of the Avastin in Lung trial. Asia Pac J Clin Oncol. 2011;7 Suppl 2:4-12. 